\title{
The effect of dornase alfa on ventilation inhomogeneity in patients with cystic
}

\section{fibrosis}

\author{
R. Amin ${ }^{\star, \#, * *}$, P. Subbarao*,\#,ף,**, W. Lou ${ }^{\#,+}$, A. Jabar*, S. Balkovec*, R. Jensen*, \\ S. Kerrigan*, P. Gustafsson ${ }^{\S, f}$ and F. Ratjen*,\#,థ
}

ABSTRACT: Outcome measures to assess therapeutic interventions in cystic fibrosis (CF) patients with mild lung disease are lacking. Our aim was to determine if the lung clearance index (LCI) can detect a treatment response to dornase alfa in paediatric CF patients with normal spirometry.

CF patients between $6-18$ yrs of age with FEV $1 \geqslant 80 \%$ pred were eligible. In a crossover design, 17 patients received 4 weeks of dornase alfa and placebo in a randomised sequence separated by a 4-week washout period. The primary end-point was the change in $\mathrm{LCl}$ from dornase alfa versus placebo. A mixed model approach incorporating period-dependent baselines was used.

The mean \pm SD age was $10.32 \pm 3.35$ yrs. Dornase alfa improved LCl versus placebo $(0.90 \pm 1.44$; $p=0.022$ ). Forced expiratory flow at $25-75 \%$ expired volume measured by $\%$ pred and $z$-scores also improved in subjects on dornase alfa $(6.1 \% \pm 10.34 \% ; p=0.03$ and $0.28 \pm 0.46 z$-score; $p=0.03)$.

Dornase alfa significantly improved $\mathrm{LCl}$. Therefore the $\mathrm{LCl}$ may be a suitable tool to assess early intervention strategies in this patient population.

KEYWORDS: Children, cystic fibrosis, dornase alfa, lung clearance index, spirometry, ventilation inhomogeneity

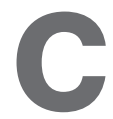
ystic fibrosis (CF) is a common fatal autosomal recessive disease. Chronic respiratory disease is responsible for the shortened life span in most patients. CF is characterised by an impairment of chloride transport resulting in impaired mucociliary clearance and recurrent pulmonary infections, which lead to irreversible structural lung damage. Dornase alfa, a recombinant human DNAse I, is an established therapeutic intervention for $\mathrm{CF}$ patients of all lung disease severities [1, 2]. Dornase alfa hydrolyses extracellular DNA present in elevated levels in lower airway secretions [3]. Dornase alfa has been shown to have a beneficial effect on lung function in patients with mild lung disease but a 2-yr study period and 474 patients were required to detect a treatment benefit [2]. Given the constant improvement in the annual decline in lung function in CF patients and the increasing proportion of CF patients with normal pulmonary function due to improved clinical care, more sensitive measures of lung function are needed for clinical studies in CF patients with mild disease.

The lung clearance index (LCI) is a measure of ventilation inhomogeneity determined during multiple breath washout (MBW). Cross-sectional studies in CF patients have shown that the LCI is more sensitive at detecting lung disease than spirometry [4-9]. LCI is determined during tidal breathing and normative values are consistent across the paediatric age range beyond infancy [4-10]. A previous trial with hypertonic saline suggested that the LCI could be a potentially useful end-point for interventional trials in $\mathrm{CF}$ patients with mild lung disease, but this needs to be confirmed by additional evidence from interventional trials in mild patients [11].

Therefore, we designed a study to test the effect of inhalation of dornase alfa in paediatric $\mathrm{CF}$ patients with a forced expiratory volume in $1 \mathrm{~s}$ (FEV1) $\geqslant 80 \%$ predicted using the LCI as the primary outcome measure. Our research hypothesis was
AFFILIATIONS

${ }^{*}$ Division of Respiratory Medicine, Dept of Paediatrics, and "Physiology and Experimental Medicine, Research Institute, The Hospital for Sick Children,

\#University of Toronto,

+Division of Biostatistics, Dalla Lana School of Public Health, Toronto, ON, Canada.

${ }^{\S}$ Dept of Paediatrics, Central Hospital, Skövde, and

${ }^{f}$ The Sahlgrenska Academy, University of Gothenburg,

Gothenburg, Sweden.

${ }^{* *}$ Both authors contributed equally.

CORRESPONDENCE

R. Amin

The Hospital for Sick Children

555 University Avenue

Toronto

ON M5G 1 X8

Canada

E-mail: reshma.amin@sickkids.ca

Received:

May 072010

Accepted after revision:

Nov 232010 
that, amongst paediatric CF patients with mild lung disease (i.e. $\mathrm{FEV} 1 \%$ pred $\geqslant 80 \%$ ), 4 weeks of dornase alfa inhalation, as compared to 4 weeks of placebo inhalation, will improve the LCI.

\section{METHODS}

\section{Participants}

Eligible patients had to have: a confirmed diagnosis of CF; be 618 yrs of age; be able to perform reproducible spirometry; have a baseline FEV1 $\geqslant 80 \%$ pred at the screening visit; and have an oxyhaemoglobin saturation of $\geqslant 90 \%$ on room air. Exclusion criteria included: airway cultures yielding Burkholderia cepacia complex in the previous 2 yrs or nontuberculous mycobacteria in the past year; current oral corticosteroid use; oxygen supplementation; lung transplantation; intravenous antibiotics or oral quinolones within 14 days of enrolment; or any investigational drugs within 30 days of enrolment.

The study was approved by the institutional review board of the Hospital for Sick Children (Toronto, ON, Canada) and by Health Canada. All tests were performed in the pulmonary lung function laboratory at the Hospital for Sick Children between March 2008 and June 2009.

\section{Study design}

This was a 12-week crossover trial consisting of two, 4-week treatment periods separated by a 4 -week washout. Study visits occurred at $0,4,8$ and 12 weeks after randomisation.

At a screening visit, demographic characteristics, clinical data, physical examination and spirometry were recorded. Eligible participants were assigned to a treatment intervention (i.e. dornase alfa in period one versus placebo in period one) by means of a concealed computer generated randomisation, performed by a research pharmacist not otherwise involved in the study. Clinicians and research personnel remained unaware of the treatment assignments throughout the study, including the primary efficacy analysis.

The solutions were indistinguishable from each other. The solutions were administered using the PARI LC $C_{\mathbb{B}}$ Star nebulizer (Pari, Midlothian, VA, USA). Patients received either $2.5 \mathrm{~mL}$ of dornase alfa or placebo once daily for each 4 -week treatment period.

At the first and third visit, spirometry and pulse oximetry were performed at baseline and $15 \mathrm{~min}$ after inhalation of the study drug. Participants whose oxyhaemoglobin saturation exceeded $90 \%$ and whose FEV1\% pred exceeded $80 \%$ of baseline value after inhalation completion were eligible to proceed in the trial. All other treatments were maintained throughout the trial.

\section{Assessment of outcomes}

LCI, spirometry and the CF questionnaire-revised (CFQ-R) were completed at each study visit [4-6, 9, 12; A. Quittner, University of Miami, Miami, FL, USA; personal communication]. LCI was performed first followed by the CFQ-R and then spirometry. LCI was the primary outcome. Secondary outcome measures included: FEV1, forced vital capacity (FVC), forced expiratory flow at $25-75 \%$ of the FVC (FEF25-75\%) and the CFQ-R.

The MBW setup was identical to the one used in previous publications by GUSTAFSSON and co-workers. [4, 9] and AURORA and co-workers $[5,6]$ with the exception of the pneumotachograph which was replaced by a Hans Rudolph pneumotachograph (Rudolph Linear Pneumotach, Hans Rudolph, Shawnee, KS, USA). Each MBW test consisted of a washin and a washout phase. All MBW tests were performed in the sitting position. Children wore nose clips and were required to breathe through a small or large mouthpiece (VacuMed mouthpieces \# 1000 and 1004; Ventura, CA, USA). We used individual mouthpieces and pneumotachs which were removed and cleaned after individual use. The decision to use the large or small mouthpiece was based on a composite measure of the patients' weight ( $\geqslant 40 \mathrm{~kg}$, large mouthpiece; $<40 \mathrm{~kg}$, small mouthpiece) and a mask visit assessed by the LCI technician during the washout. The deadspace of the small mouthpiece was $5 \mathrm{~mL}$ and the deadspace of the large mouthpiece was $8 \mathrm{~mL}$. The post-capillary deadspace was $14 \mathrm{~mL}$. Therefore, the effective deadspace was $<2 \mathrm{~mL} \cdot \mathrm{kg}^{-1}$ for all patients.

Gas concentrations were measured by mass spectrometer (AMIS 2000; Innovision A/S, Odense, Denmark). During the test, the participant was encouraged to watch a video, listen to a portable music device or read a book with the intention of distracting the subject, thus encouraging regular breathing at rest. Patients were asked to tidal breathe a dry gas mixture containing $4 \% \mathrm{SF}_{6}, 4 \% \mathrm{He}, 21 \% \mathrm{O}_{2}$ and balance $\mathrm{N}_{2}$ via a flow past system connected to the pneumotachograph until the inspiratory and expiratory $\mathrm{SF}_{6}$ concentration were equilibrated and stable at $4 \%$. Subsequently, during expiration the flow past system was disconnected and the subject was asked to tidal breathe room air. The washout phase was completed when the end tidal $\mathrm{SF}_{6}$ concentration was $<0.1 \%$ for two consecutive tidal breaths. Each washout was performed in triplicate at a minimum. The LCI was calculated as the number of lung volume turnovers (cumulative expired volume divided by the functional residual capacity (FRC)) required to reduce endtidal $\mathrm{SF}_{6}$ concentration to one 40th of the starting value [4-6]. The final LCI value represents the mean of at least three LCI manoeuvres calculated from the three technically acceptable washout tests. The following all had to be met for a washout to be considered technically acceptable: appropriate calibration of the mass spectrometer, regular tidal breathing, no evidence of leak, a plateau of $\mathrm{SF}_{6}$ concentration of $4 \%$ at the end of washing, $\mathrm{SF}_{6}$ concentration of $0 \%$ with the first inspiration during the washout phase, an acceptable capnogram and slope of washout phase on the first exhaled breath, and at least two consecutive breaths with an $\mathrm{SF}_{6}$ concentration of less than one 40th $(0.1 \%)$ the starting concentration at the end of the washout. FRC, tidal volume $(V \mathrm{~T})$ and $V \mathrm{~T} / \mathrm{FRC}$ were also measured for quality assurance.

Spirometry was performed according to American Thoracic Society standards using the Vmax systems (VIASYS, Cardinal Health, San Diego, CA, USA) [12]. Reference data was calculated using the STANOJEVIC et al. [13] reference equations. The quality of life was assessed using the CF-specific CFQ-R. One of the three participant formats of the CFQ-R was used depending on the age of the participant: adolescent and adults (patients $\geqslant 14$ yrs), children aged 12 and 13 yrs and children aged 6-11 yrs; a parent questionnaire was completed in addition where appropriate as per the CFQ-R administration guidelines (for children aged 6-13 yrs) (A. Quittner, University of Miami, Miami, FL, USA; personal communication). 
The instrument yielded a score of 0 to 100 for each domain with higher numbers indicating better function on various domains (A. Quittner, University of Miami, Miami, FL, USA; personal communication). The CFQ-R was administered prior to spirometry at each study visit.

\section{Safety and compliance}

Safety was assessed by monitoring adverse events and changes in vital signs, clinical symptoms, physical examination and/or spirometry. Patients were withdrawn from the study if they required hospital admission for a pulmonary exacerbation, corticosteroids for a new diagnosis of allergic bronchopulmonary aspergillosis or required oral fluoroquinolones during either of the two study periods. Worsening CF symptoms and/ or the prescription of antibiotics were treated as adverse events. The prescription of antibiotics was at the discretion of the responsible physician in accordance with current $\mathrm{CF}$ practice guidelines at our hospital.

The study drug was dispensed at week 0 and week 8 study visits. Used and unused ampoules were subsequently collected to assess treatment compliance at the week 4 and week 12 study visits. Compliance was based on the number of returned ampoules for each study period.

\section{Statistical analysis}

The mixed model approach was used to test the treatment effects of dornase alfa and placebo on the LCI. This method was chosen because all study participants were measured under two different conditions (dornase alfa and placebo treatments). The variables considered in the final fixed subject effects models include treatment type (dornase alfa or placebo), study period (week $0-4$ or 8-12), and perioddependent baseline measurements, following KENWARD and ROGER [14] and JONES and KENWARD [15] for obtaining unbiased treatment effects. Similar models were generated for each secondary outcome measure.

Data were analysed according to the intention-to-treat principle. Descriptive statistics were used to describe the study population. Normality was determined using the KolmogorovSmirnov test.

We calculated the sample size required for testing using dornase alfa as the main exposure variable and the LCI as the primary outcome variable. Our estimate was based on published baseline mean \pm SD LCI of $11.54 \pm 2.86$ for a population of CF school age children from the UK [5]. We estimated a treatment effect of $3.00 \pm 2.86$ in the LCI from dornase alfa versus placebo. Assuming a significance level of $5 \%$ and a power of $80 \%, 17$ study participants would be needed to complete this crossover study [16]. Based on an attrition rate of $20 \%$ from similar trials from our centre, we aimed to recruit a total of 20 patients or 17 patients that completed the protocol. p-values $<0.05$ were considered statistically significant.

Statistical Analysis Systems software version 9.2 (SAS Institute, Inc., Cary, NC, USA) was used to conduct all analyses.

\section{RESULTS}

19 patients entered the study and underwent randomisation (fig. 1 and table 1). The LCI results of one patient failed to meet the quality control criteria for one of the four study visits. One other study patient dropped out of the study after two study visits because of a pulmonary exacerbation. Complete crossover data were therefore available for 17 patients.

LCI was performed in triplicate during each testing occasion. The intra-visit coefficient of variation $(\mathrm{CV})$ was 6.8 with the upper limit of the intra-visit CV set at two times the $\mathrm{CV}$ at $13.6 \%$ (table 1 in the online supplementary material). During the course of the study, patients returned for three more visits after the first visit. The two baseline visits for each treatment period were $\sim 8$ weeks apart (mean (range) 8.3 (6.710.1) weeks); the CV for these two baseline visits was 10.3 with an upper limit of $20.6 \%$ (table 1 in the online supplementary material). The variability of the LCI was not related to the magnitude of the LCI for the two baseline visits $(r=0.22$; $\mathrm{p}=0.4$ ) (fig. 2).

LCI was measured in 28 healthy Canadian children [17]. The mean \pm SD age was $10.1 \pm 3.1$ yrs. The mean \pm SD LCI was $6.13 \pm 0.41$; the upper limit of normal based on two standard deviations is 6.95. LCI was significantly higher in our cohort of children with CF compared to aged matched healthy controls (mean difference 2.63, 95\% CI 1.96-3.31; p<0.001) [17]. If an abnormal LCI was defined as a value above $+1.96 \mathrm{SD}$ of the mean LCI from our healthy control group, all patients had abnormal LCI at the first study visit. In contrast, only three (18\%) out of 17 had abnormal FEF25-75\% z-score (fig. 1 in the online supplementary material).

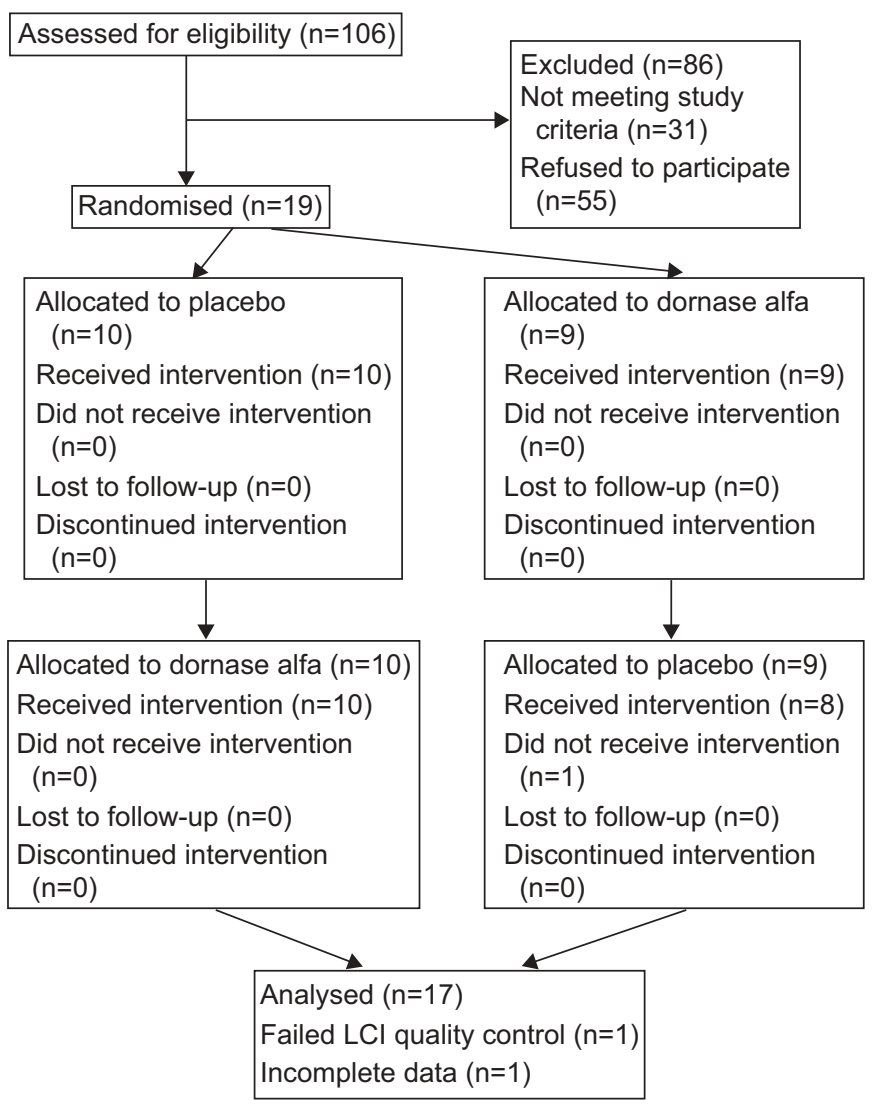

FIGURE 1. Randomisation and enrolment of study participants. LCl: lung clearance index. 


\begin{tabular}{|c|c|}
\hline & Study participants \\
\hline Subjects $\mathbf{n}$ & 17 \\
\hline Age yrs & $10.3 \pm 3.4$ \\
\hline Females/males n & $11 / 8$ \\
\hline Pseudomonas aeruginosa $+\mathrm{ve}$ & 3 \\
\hline Pancreatic insufficient \% & 95 \\
\hline$\Delta \mathrm{F} 508 / \Delta \mathrm{F} 508 \%$ & 37 \\
\hline$\Delta \mathrm{F} 508$ compound heterozygous $\%$ & 21 \\
\hline FVC \% pred & $97.6 \pm 11.4(82.5-114.1)$ \\
\hline FVC z-score & $-0.09 \pm 0.9(-0.5-0.3)$ \\
\hline FEV $1 \%$ pred & $90.7 \pm 9.1(80.00-109.78)$ \\
\hline FEV1 z-score & $-0.8 \pm 0.7(-1.1--0.5)$ \\
\hline FEV $_{1 / F V C} \%$ & $81.5 \pm 7.9(77.74-85.22)$ \\
\hline FEV1/FVC z-score & $-0.009 \pm 5.5(-2.6-2.6)$ \\
\hline FEF $25-75 \% \%$ pred & $76.9 \pm 20.0(51.7-119.2)$ \\
\hline FEF25-75\% z-score & $-1.1 \pm 0.9(-1.6--0.6)$ \\
\hline \multicolumn{2}{|c|}{$\begin{array}{l}\text { Data are presented as mean } \pm \mathrm{SD} \text { and } 95 \% \text { confidence interval, unless otherwise } \\
\text { stated. Pseudomonas aeruginosa +ve: defined as more than two positive } \\
\text { cultures in the previous year and/or currently on inhaled anti-pseudomonal } \\
\text { therapy. \% predicted spirometry values were calculated using the STANOJEVIC } \\
\text { et al. [13] reference equations. } \Delta \mathrm{F} 508 \text { : deletion of phenylalanine } 508 \text { mutation } \\
\text { of cystic fibrosis transmembrane conductance regulator; FVC: forced vital } \\
\text { capacity, FEV1: forced expiratory volume in } 1 \mathrm{~s} \text {; FEF } 25-75 \% \text { forced expiratory } \\
\text { flow at } 25-75 \% \text { of FVC; \% pred: \% predicted. }\end{array}$} \\
\hline
\end{tabular}

For $\mathrm{LCI}$, the mean $\pm \mathrm{SD}$ value at each study visit for each subject is shown in table 2 in the online supplementary material. Initial analysis of the data revealed a period effect due to the fact that the baselines were not equal; LCI was significantly worse prior to placebo therapy than dornase alfa, $8.75 \pm 1.72$ and $8.31 \pm 1.48$, respectively $(p=0.034)$. Thus, a mixed model analysis with fixed subject effects was performed with baselines as a factor which determines the relative improvement in LCI from dornase alfa compared with placebo. In this model, 4 weeks of dornase alfa resulted in a significantly improved LCI compared with placebo $(0.9 \pm 0.34 ; \mathrm{p}=0.02)$ (table 2$)$. The spectrum of treatment response to dornase alfa and placebo is shown in figure 3. There were no significant differences in FRC (L), VT (L) or $V \mathrm{~T} / \mathrm{FRC}(\%)$ for either placebo or dornase alfa treatment periods.

The absolute changes for LCI from placebo and dornase alfa inhalations are presented in table 2 in the online supplementary material. The mean \pm SD $(95 \% \mathrm{CI})$ for absolute change in LCI from placebo was $0.31 \pm 1.36(-0.33-0.95)$. The mean \pm SD (95\% CI) for absolute change in LCI from dornase alfa was $0.71 \pm 1.23(0.12-1.3)$. The mean \pm SD $(95 \% \mathrm{CI})$ for the mean difference for the pooled placebo data for this study and the hypertonic saline study was $0.069 \pm 1.16(-2.25-2.39)$ [11]. The inter-visit CV for the pooled placebo data for both studies was $7.24 \%$ with the upper limit of the inter-visit coefficient set at two times the CV or $14.5 \%$. Therefore, based on these two different methodologies, three patients and four patients had improvements in the LCI that fell above the upper limit of the $95 \% \mathrm{CI}$ and two times the inter-visit $\mathrm{CV}$, respectively.

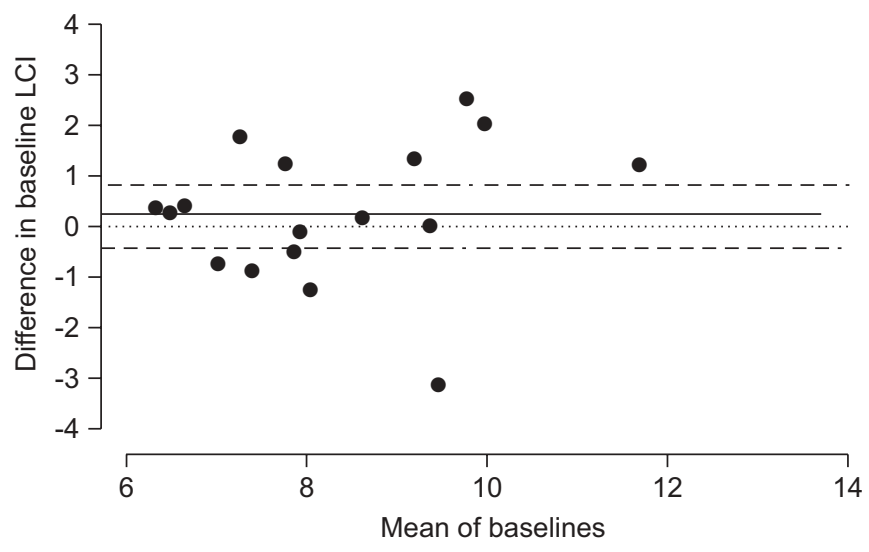

FIGURE 2. The lung clearance index (LCI) limits of agreement for the mean of the two baseline LCI measurements versus the difference in $\mathrm{LCl}$ of the two baseline measurements. - : mean; ----: 95\% confidence interval.

For $\mathrm{FEF} 25-75 \% \%$ pred, the mean \pm SD values for each study visit for each subject is shown in table 2 in the online supplementary material and in figure 4. Small airway flows as measured by FEF25-75\% in $\mathrm{L} \cdot \mathrm{s}^{-1}, \%$ pred and $\mathrm{z}$-scores also improved in subjects on dornase alfa compared with those on placebo $\left(0.18 \pm 0.72 \mathrm{~L} \cdot \mathrm{s}^{-1}\right.$; $\mathrm{p}=0.03 ; 6.1 \pm 10.34 \%$ pred; $\mathrm{p}=0.03$; and $0.28 \pm 0.46 \mathrm{z}$-score; $\mathrm{p}=0.03$ ) in the fixed subject effects model (table 2).

FEV1 and FVC in \% pred, $\mathrm{L} \cdot \mathrm{s}^{-1}$ and z-scores as well as CFQ-R respiratory domain and $C F Q-R$ parent respiratory domain scores were not significantly different after dornase alfa inhalation compared with placebo (table 2 and fig. 2 in the online supplementary material). LCI significantly correlated with FEF25-75\% \% pred, FEF25-75\% z-score, FEF25-75\% L $\cdot \mathrm{s}^{-1}$, FEV1 \% pred and FEV1 z-score. The strongest correlation was found between LCI and FEF25-75\% \% pred $(r=-0.43 ; p=0.0001)$ (fig. 5 and table 3 in the online supplementary material).

After administration of the first dose of assigned solution, the FEV1 fell by a mean \pm SD of $1 \pm 135 \mathrm{~mL} \cdot \mathrm{s}^{-1}$ after placebo inhalation and $39 \pm 140 \mathrm{~mL} \cdot \mathrm{s}^{-1}$ after dornase alfa inhalation, but there was no significant difference $(p=0.47)$. None of the patients had a drop of FEV1 \% pred $\geqslant 20 \%$. There were more overall adverse events during the dornase alfa treatment period as compared with the placebo period (table 4 in the online supplementary material). Adverse events included cough, pulmonary exacerbation, increased sputum production, rhinorrhoea, dry throat, sore throat, wheeze and chest pain. Cough was the most common adverse event and was experienced by seven patients during the dornase alfa treatment periods.

Adherence to treatment, as judged by the number of returned ampoules was $88.93 \pm 13.42 \%$ for the placebo study period and $95.81 \pm 4.79 \%$ for the dornase alfa study period $(\mathrm{p}=0.043)$. $>80 \%$ compliance was seen in $79 \%$ of patients during the placebo treatment and $100 \%$ of patients during treatment with dornase alfa. Treatment allocation sequence was correctly guessed by nine $(47 \%)$ out of 19 of the study participants.

\section{DISCUSSION}

We have demonstrated that the LCI, as well as FEF25-75\% $\mathrm{Z}$-score, \% pred and $\mathrm{L} \cdot \mathrm{s}^{-1}$ is able to detect a treatment effect from 28 days of dornase alfa as compared with placebo in $17 \mathrm{CF}$ 
TABLE 2 Summary of outcome measures using the mixed model approach with fixed subject effects

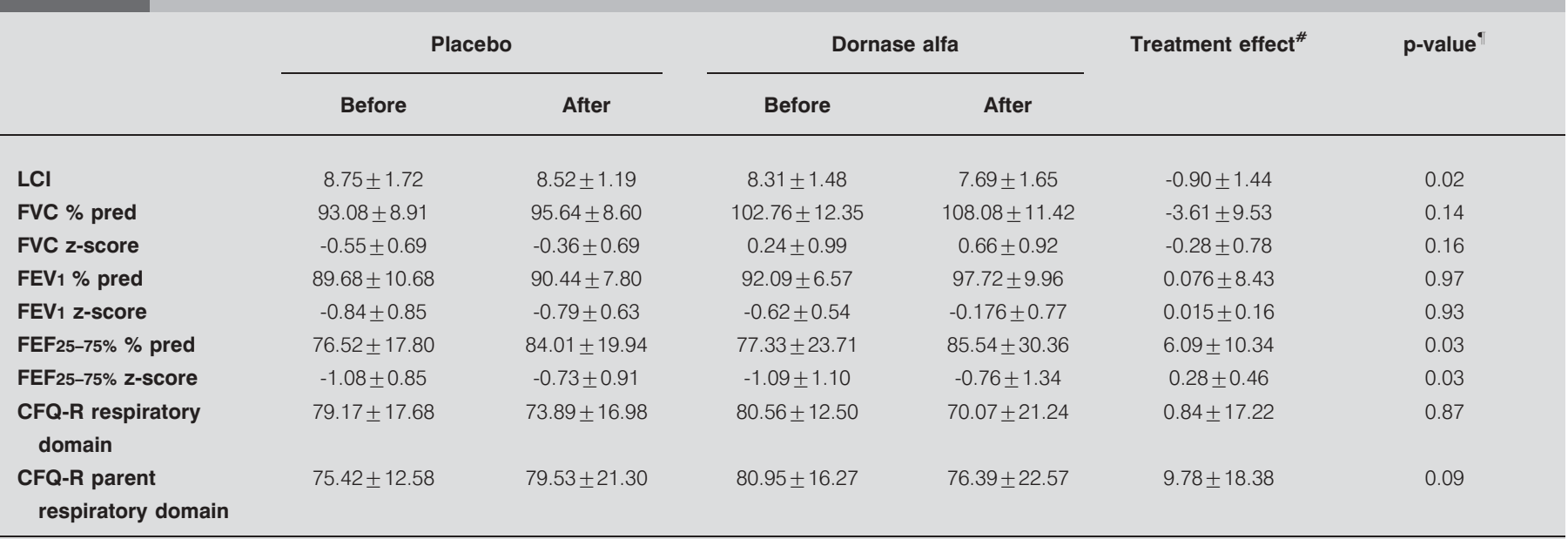

Data are presented as mean \pm SD, unless otherwise stated. \% predicted spirometry values were calculated using the STANOJEVIC et al. [13] reference equations. LCI: lung clearance index; FVC: forced vital capacity; FEV1: forced expiratory volume in 1 s; FEF25-75\%: forced expiratory flow at 25-75\% of FVC; \% pred: \% predicted; CFQ-R: cystic fibrosis questionnaire-revised. "\#: estimated treatment effects (placebo versus dornase alfa) from the model; ": p-value for comparison of post-placebo to post-dornase alfa, while incorporating the period-dependent baseline (pre) measures.

patients with mild lung disease. These data add to the evidence that LCI may be a potential outcome measure for interventional trials in patients with preserved lung function.

Although the results regarding the effects on LCI are similar to our recent findings regarding hypertonic saline in a similar patient population, there are some notable differences [11]. The magnitude of the treatment effect of the LCI from dornase alfa was smaller than for hypertonic saline. This is surprising given the published literature suggesting the contrary with regards to lung function [18]. In this study the FEF25-75\% parameters also significantly improved with dornase alfa in addition to the LCI, thus supporting the LCI as a measure of the small airways. However, there was no significant correlation between the changes in these two parameters during treatment, suggesting

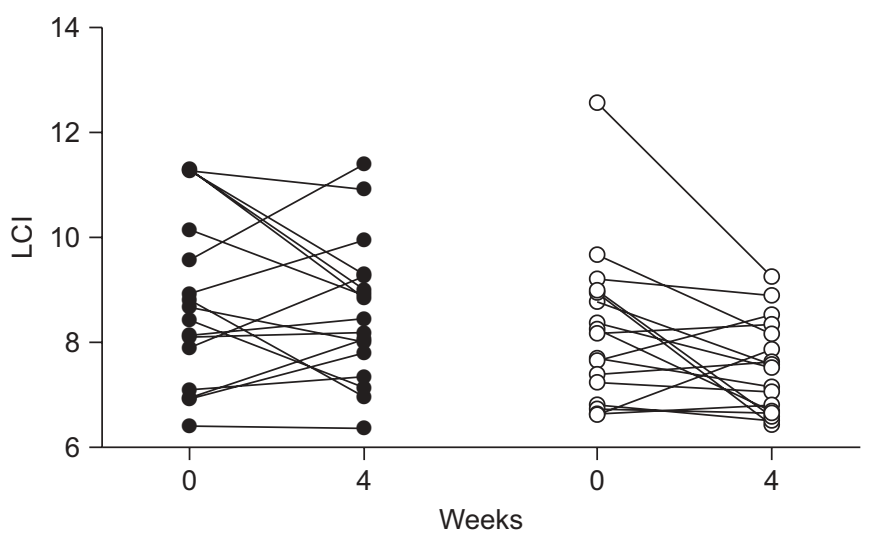

FIGURE 3. Pre- and post-treatment lung clearance index (LCl) for each study participant for the placebo treatment period $(\bullet)$ and the dornase alfa treatment period $(\bigcirc)$. $p=0.022$. The $p$-value represents the difference in the treatment effect size of dornase alfa versus placebo calculated using the mixed model approach with fixed subject effects. that complementary information can be obtained by performing both tests, as previously shown in a cross-sectional study of the LCI and raised lung volume rapid thoraco-abdominal compression in CF infants [10].

Another possible explanation may lie in the different mechanism of action of each of these drugs. Dornase alfa is a mucolytic agent that decreases the viscosity and surface adhesion properties of CF sputum and thus improves mucus clearance [3]. However, hypertonic saline improves mucociliary clearance by restoring the depleted airway surface liquid layer. Therefore, the treatment responsiveness of LCI could differ in sensitivity depending on the mechanism of action of the intervention. This raises the question whether LCI may be particularly sensitive to interventions that improve airway

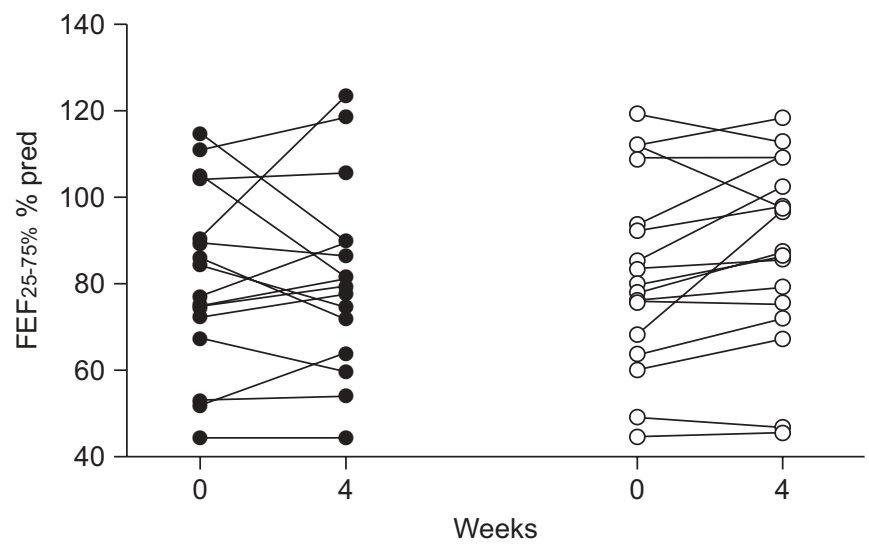

FIGURE 4. Pre- and post-treatment forced expiratory flow at $25-75 \%$ of forced vital capacity $\left(\mathrm{FEF}_{25}-75 \%\right)$ for each study participant for the placebo treatment period $(\bullet)$ and the dornase alfa treatment period $(\bigcirc) . p=0.029$. The $p$-value represents the difference in the treatment effect size of dornase alfa versus placebo calculated using the mixed model approach with fixed subject effects. 


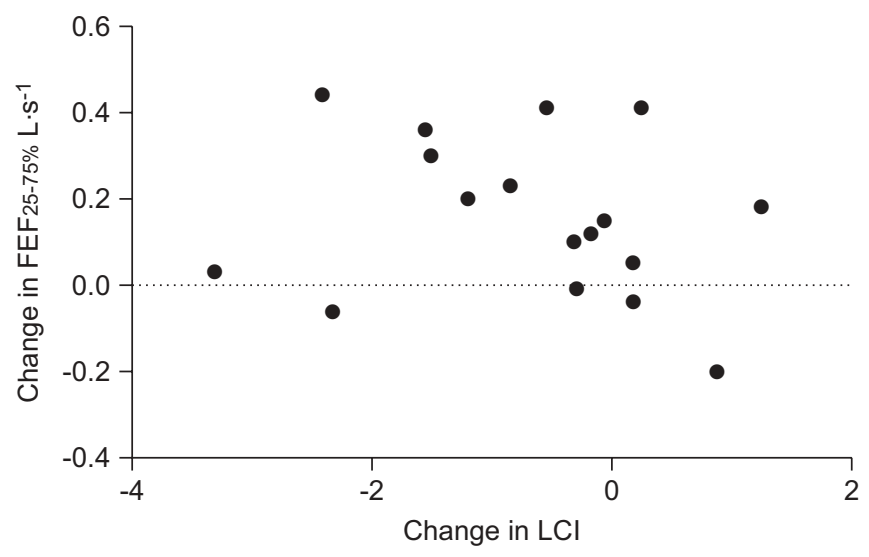

FIGURE 5. Correlation between the change in the lung clearance index $(\mathrm{LCl})$ and the change in forced expiratory flow at $25-75 \%$ of forced vital capacity (FEF25-75\%) from 4 weeks of dornase alfa inhalation.

surface hydration and mucociliary clearance, an early step of CF pathophysiology. If confirmed this could potentially have implications for interventional studies of agents targeting the basic defect, such as gene replacement therapy, cystic fibrosis transmembrane conductance regulator (CFTR) or non-CFTR directed ion channel pharmacotherapy [19].

Finally, the differences between the two interventional studies may be explained by differing changes in the placebo group. In this study, LCI trended towards improvement during the placebo period (regardless of treatment sequence) while it worsened in the hypertonic saline study [11]. Therefore, given the crossover design of both trials, the different magnitudes of the treatment effect may have been driven by the placebo effects going in opposite directions. As placebo effects are difficult to predict in study designs it is reassuring that LCI was able to detect a treatment effect in both studies, regardless of the behaviour of the placebo group. In addition, when the placebo arm is excluded, the magnitude of the treatment effect from dornase alfa and hypertonic saline (excluding the placebo periods) as measured by the LCI are not different $(0.71 \pm 0.30$ versus $0.88 \pm 0.29 ; \mathrm{p}=0.99$ ).

There were several limitations of the current study. The mean treatment effect and variability of the LCI from dornase alfa was less than what was anticipated in the sample size calculation a priori. The reason for this has been previously explained elsewhere [11]. Furthermore, the baseline LCI measurements before placebo and dornase alfa treatment periods were significantly different. This probably reflects chance variation and the ability of the LCI to measure fluctuations in lung disease in CF patients over time, thus supporting the robustness of the LCI as a primary outcome measure. Our study was limited to a patient population that was able to perform technically adequate spirometry and thus it has not yet been established as to whether the LCI is a responsive outcome measure for a treatment intervention trial in infants and preschool children. In addition, type 1 error is always appreciable when making conclusions based on small numbers of patients. Therefore, there is a $5 \%$ chance that this is the case. Based on our study results, there was a $1 \%$ chance of type 2 error.
In summary, the LCI is a sensitive and responsive outcome measure that was able to detect a significant treatment effect from dornase alfa in a paediatric cohort of CF patients with mild lung disease. Although there is some overlap, the LCI and the forced expiratory flow parameters seem to represent different structural and functional abnormalities of the small airways and, subsequently, identify different treatment responders. Therefore, the LCI is a promising outcome measure given the ability to perform clinical testing in patients of all ages and disease severities, to identify treatment responders that would not be identified by other conventional outcomes and its potential to facilitate the rapid integration of new therapies into clinical practice.

\section{SUPPORT STATEMENT}

Financial support for this study has been provided by Roche Canada and the Lynn and Arnold Irwin Foundation.

\section{CLINICAL TRIAL}

This study was registered with www.ClinicalTrials.gov, number NCT00557089.

\section{STATEMENT OF INTEREST}

A statement of interest for F. Ratjen and the study itself can be found at www.erj.ersjournals.com/site/misc/statements.xhtml

\section{REFERENCES}

1 Fuchs HJ, Borowitz DS, Christiansen DH, et al. Effect of aerosolized recombinant DNAse on exacerbations of respiratory symptoms and on pulmonary function in patients with cystic fibrosis. N Engl J Med 1994; 331: 637-642.

2 Quan JM, Tiddens H, Sy J, et al. A two-year randomized, placebocontrolled trial of dornase alfa in young patients with cystic fibrosis with mild lung function abnormalities. J Pediatr 2001; 139: 813-820.

3 Shak S, Capon DJ, Hellmiss R, et al. Recombinant human DNAse I reduces the viscosity of cystic fibrosis sputum. Proc Natl Acad Sci USA 1990; 87: 9188-9192.

4 Gustafsson PM, Aurora P, Lindblad A. Evaluation of ventilation maldistribution as an early indicator of lung disease in children with cystic fibrosis. Eur Respir J 2003; 22: 972-979.

5 Aurora P, Gustafsson P, Bush A, et al. Multiple breath inert gas washout as a measure of ventilation distribution in children with cystic fibrosis. Thorax 2004; 59: 1068-1073.

6 Aurora P, Bush A, Gustafsson P, et al. Multiple-breath washout as a marker of lung disease in preschool children with cystic fibrosis. Am J Respir Crit Care Med 2005; 171: 249-256.

7 Kraemer R, Blum A, Schibler A, et al. Ventilation inhomogeneities in relation to standard lung function in patients with cystic fibrosis. Am J Respir Crit Care Med 2005; 171: 371-378.

8 Horsley AR, Gustafsson PM, Macleod KA, et al. Lung clearance index is a sensitive, repeatable and practical measure of airways disease in adults with cystic fibrosis. Thorax 2008; 63: 135-140.

9 Gustafsson PM, De Jong PA, Tiddens HA, et al. Multiple-breath inert gas washout and spirometry versus structural lung disease in cystic fibrosis. Thorax 2008; 63: 129-134.

10 Lum S, Gustafsson P, Ljungberg H, et al. Early detection of cystic fibrosis lung disease: multiple-breath washout versus raised volume tests. Thorax 2007; 62: 341-347.

11 Amin R, Subbarao P, Jabar A, et al. Hypertonic saline improves the LCI in paediatric CF patients with normal lung function. Thorax 2010; 65: 379-383.

12 Miller MR, Hankinson J, Brusasco V, et al. Standardisation of spirometry. Eur Respir J 2005; 26: 319-338. 
13 Stanojevic S, Wade A, Stocks J, et al. Reference ranges for spirometry across all ages: a new approach. Am J Respir Crit Care Med 2008; 177: 253-260.

14 Kenward MG, Roger JH. The use of baseline covariates in crossover studies. Biostatistics 2010; 11: 1-17.

15 Jones B, Kenward MG. Design and Analysis of Cross-over Trials. 2nd Edn. London, Chapman \& Hall/CRC, 2003; pp. 206-213.

16 Schoenfeld DA. Statistical consideration for clinical trials and scientific experiments. http://hedwig.mgh.harvard.edu/sample_ size/size.html Date last accessed: February 1, 2010. Date last updated: May 14, 2010.
17 Brown M, Amin R, Ratjen F, et al. Evaluation of lung clearance index in children with cystic fibrosis compared to Canadian controls. Am J Respir Crit Care Med 2010; 181: A6815.

18 Suri R, Metcalfe C, Lees B, et al. Comparison of hypertonic saline and alternate-day or daily recombinant human deoxyribonuclease in children with cystic fibrosis: a randomised trial. Lancet 2001; 358: 1316-1321.

19 Alton E, Davies JC, Griesenbach U, et al. Single dose of pGM169/ GL67A in CF patients. http://clinicaltrials.gov/ct2/show/ NCT00789867 Date last accessed: January 17, 2010. Date last updated: August 13, 2009. 